\title{
Ambrisentan for the treatment of pulmonary arterial hypertension: improving outcomes
}

This article was published in the following Dove Press journal:

Patient Preference and Adherence

7 May 2013

Number of times this article has been viewed

\author{
Soha M Elshaboury' \\ Joe R Anderson ${ }^{2}$ \\ 'College of Pharmacy, University \\ of New Mexico, ${ }^{2}$ Pharmacy Practice \\ and Internal Medicine, College of \\ Pharmacy and School of Medicine, \\ University of New Mexico, \\ Albuquerque, NM, USA
}

\begin{abstract}
Pulmonary arterial hypertension (PAH) is a progressive disease of the pulmonary vasculature that is associated with severe functional impairment and a poor prognosis. Ambrisentan is a selective endothelin type A receptor antagonist approved for the treatment of patients with PAH World Health Organization group 1. The efficacy and safety of ambrisentan has been evaluated in the ARIES series (Ambrisentan for the Treatment of Pulmonary Arterial Hypertension, Randomized, Double-Blind, Placebo-Controlled, Multicenter, Efficacy Studies), which has established its use as both monotherapy or in conjunction with other PAH therapies. Specifically, ambrisentan is effective at increasing exercise tolerance, decreasing the risk of functional class deterioration, and prolonging time to clinical worsening. Further, ambrisentan has a favorable effect on mortality, with an $88 \%$ patient survival rate after two years of therapy compared with a $61 \%$ survival rate as estimated by the National Institute of Health Registry. Ambrisentan is generally well tolerated in all patient groups, with the main side effects of peripheral edema, sinusitis, flushing, and nasal congestion considered to be mild to moderate in nature. Ambrisentan has several favorable qualities that potentially make it more acceptable to patients, including once-daily administration, limited adverse drug reactions and drug-drug interactions, and minimal risk of liver enzyme elevation. Because of the potential risk of teratogenicity associated with ambrisentan, it is only available through a limited distribution program, ie, LEAP (the Letairis Education and Access Program). Ongoing clinical trials will help to clarify the role of ambrisentan in the treatment of PAH.
\end{abstract}

Keywords: ambrisentan, endothelin, endothelin receptor antagonist, pulmonary arterial hypertension, Letairis Education and Access Program

\section{Background}

Pulmonary arterial hypertension (PAH) is a unique and progressively debilitating disease that is characterized by elevated pulmonary arterial pressure secondary to vasoconstriction, hypertrophy, and remodeling of the pulmonary vasculature. PAH is associated with severe functional impairment and a poor prognosis, with a median survival of 2.8 years in untreated patients. ${ }^{1}$ The cause of death in the majority of patients is either right-sided heart failure $(47 \%)$ or sudden cardiac death $(26 \%){ }^{1}$

The most frequent presenting symptom of PAH is dyspnea on exertion $(60 \%$ of patients), which may progress to dyspnea at rest as the disease advances. ${ }^{2}$ Other common complaints include fatigue, weakness, exercise intolerance, anginal chest pain, and syncope. ${ }^{2}$ The initial screening process for PAH should begin with a comprehensive family history, medical history, and physical examination. ${ }^{2}$ Guidelines recommend an echocardiogram initially to evaluate for the presence of $\mathrm{PAH}^{2}$ If present, the
Correspondence: Joe R Anderson College of Pharmacy, University of New Mexico, MSC 09 5360, Albuquerque, NM 87I3I-000I, USA Email janderson@salud.unm.edu 
echocardiogram can be used to determine if the PAH is due to congenital or left heart disease. Additional tests (human immunodeficiency virus and antinuclear antibody serology, ventilation/perfusion scan, pulmonary function tests, overnight oximetry, liver function tests) should be done to determine the etiology of suspected $\mathrm{PAH}{ }^{2}$ Confirmation of the diagnosis of $\mathrm{PAH}$ requires right heart catheterization, which is also used to determine disease severity and to guide treatment decisions. ${ }^{2}$

PAH is classified by the World Health Organization (WHO) according to etiology. ${ }^{3}$ WHO group $1 \mathrm{PAH}$ is the most prevalent, and includes $\mathrm{PAH}$ associated with idiopathic etiology, genetic predisposition, congenital heart abnormalities, connective tissue disorder, portal hypertension, use of anorexigen, and human immunodeficiency virus. ${ }^{3}$ WHO groups 2-5 refer to PAH associated with left heart disease, lung disease, chronic thromboembolic disease, and miscellaneous causes, respectively. ${ }^{3}$

Progression and prognosis of PAH is nonlinear. Clinical status and severity are classified by the WHO functional classification scale. ${ }^{4}$ Functional classification I represents patients with the least restrictive symptoms (asymptomatic) on ordinary activities of daily living, while functional classification IV describes the most functionally impaired patients, in whom symptoms occur at rest. ${ }^{4}$ Functional classifications II and III refer to patients with slight limitations on activities of daily living and those with marked limitations on activities of daily living, respectively. ${ }^{4}$ WHO functional classification I and II are associated with the best prognosis, and have a median predicted survival of 58 months; class III has a less favorable prognosis of 36 months; and class IV is associated with the worst prognosis, having a median survival of 6 months. ${ }^{1}$

The pathogenesis of PAH is the result of an imbalance between key mediators in the pulmonary circulation. This imbalance leads to increased thrombosis, vasoconstriction, and proliferation of smooth muscle and endothelial cells within the pulmonary vasculature. ${ }^{5}$ Among these mediators is endothelin-1, a potent vasoconstrictor that also stimulates the proliferation of smooth muscle cells. ${ }^{6}$ Levels of endothelin-1 have been established to be elevated in patients with $\mathrm{PAH}$ and contribute to progression of the disease. This observed increase in endothelin-1 levels is correlated with a proportional decrease in pulmonary blood flow and cardiac output. ${ }^{5}$ As a result, therapies that block the detrimental effects of endothelin-1 have been recently developed.

Endothelin receptor antagonists represent a drug class currently being utilized in the management of PAH.
Ambrisentan (Letairis $^{\circledR}$, Gilead Sciences Inc, Foster City, CA, USA) was approved by the US Food and Drug Administration (FDA) in 2007 for the treatment of primary $\mathrm{PAH}$ (WHO group 1). The purpose of this review is to assess the efficacy, safety, and patient acceptability of ambrisentan in the treatment of PAH.

\section{Pharmacology}

Ambrisentan is a propanoic acid, nonsulfonamide, selective endothelin type $\mathrm{A}\left(\mathrm{ET}_{\mathrm{A}}\right)$ receptor antagonist. The endothelin neurohormonal system is comprised of three isoforms, ie, endothelin-1, endothelin-2, and endothelin-3, each consisting of 21 amino acid residues. ${ }^{7}$ The most abundant and predominant isoform is endothelin- $1 .{ }^{8}$ Endothelin- 1 has potent vasoconstrictor activity as well as proliferative and hypertrophic actions. ${ }^{8}$ Levels of endothelin- 1 have been demonstrated to be elevated in patients with PAH and contribute to progression of the disease. Endothelin acts via two specific receptor subtypes, $\mathrm{ET}_{\mathrm{A}}$ and $\mathrm{ET}_{\mathrm{B}}$, which have different pharmacologic functions. ${ }^{9} \mathrm{ET}_{\mathrm{A}}$ receptors mediate the vasoconstrictive, proliferative, and hypertrophic effects of endothelin-1., ${ }^{8,10,11} \mathrm{ET}_{\mathrm{B}}$ receptors promote vasodilation via release of nitric oxide or prostacyclin, and mediates the pulmonary clearance of circulating endothelin-1. ${ }^{12}$ In the human lung, $\mathrm{ET}_{\mathrm{A}}$ is the dominant endothelin receptor subtype, existing in a ratio of 93:7 compared with the $\mathrm{ET}_{\mathrm{B}}$ subtype. ${ }^{13}$

Given the activity and location of the $\mathrm{ET}_{\mathrm{A}}$ receptor, therapy has been targeted towards blocking the effect of endothelin on the $\mathrm{ET}_{\mathrm{A}}$ receptor, utilizing either the nonselective $\mathrm{ET}_{\mathrm{A}} / \mathrm{ET}_{\mathrm{B}}$ receptor antagonist, bosentan, or the selective $\mathrm{ET}_{\mathrm{A}}$ receptor antagonist, ambrisentan (Table 1). A previous selective endothelin receptor antagonist, sitaxsentan, was approved for treatment of PAH in the European Union and Canada, but was removed from the market as a result of idiosyncratic hepatotoxicity. ${ }^{14}$ There are theoretical benefits for using a selective $\mathrm{ET}_{\mathrm{A}}$ receptor antagonist, which include blocking the vasoconstrictive and proliferative effects of endothelin-1 at the $\mathrm{ET}_{\mathrm{A}}$ receptor, while at the same time preserving the potentially beneficial effect of endothelin-1 activation at the $\mathrm{ET}_{\mathrm{B}}$ receptor (vasodilation and enhanced clearance of endothelin-1). Which type of endothelin-1 antagonist is preferred is debatable, because there are currently no head-to-head clinical trials comparing the two therapies; however, both agents have documented clinical benefits in the treatment of PAH. ${ }^{15}$

As mentioned, ambrisentan is a selective antagonist of the $\mathrm{ET}_{\mathrm{A}}$ receptor, with an affinity of $>4000: 1$ as compared with the $\mathrm{ET}_{\mathrm{B}}$ receptor. ${ }^{16,17}$ Administration of ambrisentan 
Table I Pharmacologic and pharmacokinetic properties of endothelin receptor antagonists ${ }^{16,17,31}$

\begin{tabular}{|c|c|c|c|c|c|}
\hline $\begin{array}{l}\mathrm{ET}_{\mathrm{A}}: \mathrm{ET}_{\mathrm{B}} \text { receptor } \\
\text { selectivity }\end{array}$ & $\mathbf{T}_{\max }$ & Metabolism & $\begin{array}{l}\text { Elimination } \\
\text { half-life }\end{array}$ & Dosing & Drug interactions \\
\hline \multicolumn{6}{|l|}{ Bosentan } \\
\hline $240-360: 1$ & 3.5 hours & $\begin{array}{l}\text { Hepatic (CYP3A4, } \\
\text { CYP2C9) }\end{array}$ & 5.4 hours & $\begin{array}{l}62.5 \mathrm{mg} \text { BID for } \\
4 \text { weeks, then } 125 \mathrm{mg} \\
\text { BID (if }<40 \mathrm{~kg} \text {, } \\
62.5 \mathrm{mg} \text { BID) }\end{array}$ & $\begin{array}{l}\text { Moderate and strong inhibitors of CYP3A4 } \\
\text { and CYP2C9 increase bosentan; } \\
\text { substrates of CYP3A4 } 4 \text { and CYP2C9 } \\
\text { concentrations decrease due to induction } \\
\text { by bosentan }\end{array}$ \\
\hline \multicolumn{6}{|l|}{ Ambrisentan } \\
\hline$>4000: 1$ & 2 hours & $\begin{array}{l}\text { Hepatic (CYP3A4, } \\
\text { CYP2CI9, } \\
\text { UGT IA9S, } \\
\text { 2B7S, IA3S) }\end{array}$ & 9 hours & $\begin{array}{l}5 \mathrm{mg} \text { once daily } \\
\text { titrated to } 10 \mathrm{mg} \\
\text { if tolerated }\end{array}$ & $\begin{array}{l}\text { Does not inhibit or induce CYP enzymes; } \\
\text { cyclosporine increased ambrisentan } \\
\text { concentrations by two-fold }\end{array}$ \\
\hline
\end{tabular}

Abbreviations: $\mathrm{T}_{\max }$, time to maximal concentration; CYP, cytochrome P450; ET, endothelin; UGT, uridine 5'-diphosphate glucuronosyltransferase; BID, twice a day.

in patients with PAH is associated with vascular smooth muscle relaxation and vasodilation, as evidenced by a reduction in pulmonary vascular resistance and mean arterial pressure. ${ }^{18}$

\section{Pharmacokinetics}

Ambrisentan is rapidly absorbed, with peak concentrations achieved at two hours post administration, and administration with food has no effect on its bioavailability. ${ }^{16,17}$ Ambrisentan undergoes intestinal extrusion through the P-glycoprotein system, is highly protein-bound (99\%), and is eliminated predominantly by nonrenal mechanisms, with $<5 \%$ excreted unchanged in the urine. ${ }^{16,17}$ Therefore, the dose of ambrisentan does not need adjustment in individuals with mild to moderate renal impairment. ${ }^{16}$ There are currently no data on the use of ambrisentan in patients with severe renal impairment or those receiving hemodialysis. Ambrisentan is metabolized by cytochrome P450 (CYP)3A4 and CYP2C1, as well as by the uridine $5^{\prime}$-diphosphate glucuronosyltransferases, 1A9S, 2B7S, and $1 \mathrm{~A} 3 \mathrm{~S} .{ }^{16,17}$ In addition, in vitro studies indicate that ambrisentan is a substrate for the organic anion transport polypeptides $1 \mathrm{~B} 1$ and $1 \mathrm{~B} 3 .{ }^{16}$ However, the only documented significant drug interaction is with cyclosporine, for which coadministration resulted in a respective 1.5-fold and 2-fold increase in the peak concentration and area under the concentration-time curve for ambrisentan. As a result, ambrisentan dosing should not exceed $5 \mathrm{mg}$ per day if coadministered with cyclosporine. Ambrisentan does not inhibit or induce hepatic drug metabolism pathways. Specific to the treatment of PAH, ambrisentan does not interact with sildenafil, tadalafil, warfarin, or digoxin. The elimination half-life of ambrisentan is 15 hours, although the mean trough concentration at steady-state with once-daily dosing is only $15 \%$ of the mean peak concentration. ${ }^{16,17}$ However, no studies have examined the clinical effects at trough concentration nor the effect of twice-daily dosing.

\section{Availability}

Ambrisentan is marketed as Letairis ${ }^{\circledR}$ in $5 \mathrm{mg}$ and $10 \mathrm{mg}$ tablets. ${ }^{16}$ Because of the potential risk of teratogenicity, ambrisentan is only available through the Letairis Education and Access Program (LEAP) ${ }^{19}$ which is a Risk Evaluation and Mitigation Strategies program designed to eliminate the risk of fetal abnormalities and/or death in females of childbearing potential receiving ambrisentan. A female of childbearing potential is defined by LEAP as a nonmenopausal female who has not had a hysterectomy, bilateral oophorectomy, or medically documented ovarian failure. ${ }^{19}$ This includes pubertal females who have not yet had a menses (Tanner stage 3), perimenopausal women who have had a spontaneous menses in the last 12 months, and women who have undergone a tubal sterilization. ${ }^{19}$

The program stipulates that all female patients of childbearing potential must undergo an initial pregnancy test to rule out gravidity prior to the start of therapy. ${ }^{19}$ Furthermore, these patients must undergo monthly pregnancy testing to rule out pregnancy in order to continue receiving ambrisentan. In addition, females of childbearing potential must consent to using two methods of contraception throughout the duration of treatment, as well as for one month after discontinuation of treatment. Patients who decide to use one of the following methods for pregnancy prevention are exempt from using an additional method of contraception: a copper T 380A or long-acting levonorgestrel intrauterine device, or tubal sterilization.

All ambrisentan prescriptions must be obtained from a physician registered with the LEAP program and who has signed the LEAP prescriber enrollment and agreement form. ${ }^{19}$ 
By enrolling in the program, the physician acknowledges that he/she has read the full prescribing information for ambrisentan, understands the risks and benefits associated with therapy, agrees to monitor patients appropriately, and agrees to report significant adverse events to LEAP. The physician must also acknowledge that it is their obligation to educate the patient regarding the risks of therapy and appropriate risk reduction strategies.

Ambrisentan can only be dispensed by a certified specialty pharmacy registered with LEAP. ${ }^{19}$ The pharmacy is responsible for contacting all female patients of childbearing potential each month to confirm completion of pregnancy testing, counsel on the risk of teratogenicity, and answer any patient questions prior to mailing the prescription.

Ambrisentan is designated by the FDA as an orphan drug because it is approved for treatment of a disease that affects fewer than 200,000 people in the United States. The monthly cost of ambrisentan therapy is US\$2550, with most insurance companies covering prescription costs at different thresholds. ${ }^{20} \mathrm{~A}$ study evaluating patient access to ambrisentan, as well as other orphan drugs, found accessibility to be seriously limited by the high cost of therapy, even in the setting of prescription insurance. ${ }^{20}$

\section{Assessment of efficacy}

The efficacy and safety of ambrisentan has been evaluated in the Ambrisentan for the Treatment of Pulmonary Arterial Hypertension, Randomized, Double-Blind, PlaceboControlled, Multicenter, Efficacy Studies (ARIES). ${ }^{21,25,27}$

\section{ARIES-I and ARIES-2}

The Phase III ARIES-1 and ARIES-2 trials were conducted concurrently to evaluate the efficacy and tolerability of different doses of ambrisentan in patients with group $1 \mathrm{PAH} .{ }^{21}$ A total of 202 and 192 patients were enrolled in the ARIES-1 and ARIES-2 studies, respectively, between December 2003 and February 2006.

Patients were eligible for enrollment in the study if they had a diagnosis of group $1 \mathrm{PAH}$ and were excluded from participation if they were receiving additional treatment for PAH (bosentan, sitaxsentan, sildenafil, epoprostenol, iloprost, or treprostinil), or if they had a baseline six-minute walk distance test (6MWD) of less than 150 meters (m) or greater than $450 \mathrm{~m} \cdot{ }^{21}$ Patients enrolled in the study were predominantly Caucasian (76\%), female (78\%), and classified as WHO functional class II $(38 \%)$ or III $(55 \%)$.

Patients were randomized in a $1: 1: 1$ ratio to receive once-daily ambrisentan $5 \mathrm{mg}, 10 \mathrm{mg}$, or placebo (ARIES-1) or ambrisentan $2.5 \mathrm{mg}, 5 \mathrm{mg}$, or placebo (ARIES-2) for 12 weeks. ${ }^{21}$ The primary endpoint, functional exercise capacity as measured by the $6 \mathrm{MWD}$, was assessed at baseline, and at 4,8 , and 12 weeks. Several secondary outcomes were also evaluated for changes from baseline, including: time to clinical worsening, WHO functional classification, plasma B-type natriuretic peptide levels, and patient quality of life using the Short Form-36 Health Survey Physical Functioning scale.

An increase in the 6MWD was reported in the ambrisentan group as early as week 4 , with a significant increase in exercise capacity at week $12 .{ }^{21}$ Placebo-corrected increases in the $6 \mathrm{MWD}$ at 12 weeks were $32 \mathrm{~m}$ (95\% confidence interval [CI] 2-63; $P=0.022), 45 \mathrm{~m}(95 \% \mathrm{CI} 24-65 ; P<0.001)$, and $51 \mathrm{~m}(95 \%$ CI $27-76 ; P<0.001)$ in the $2.5 \mathrm{mg}, 5 \mathrm{mg}$, and $10 \mathrm{mg}$ dose groups, respectively.

It is important to note that the 6MWD is a measure of functional exercise capacity that is only moderately correlated with improvements in quality of life and mortality risk. ${ }^{22}$ Further, a statistically significant change in the 6 MWD may not be equivalent to a clinically significant change. ${ }^{22}$ Use of the 6 MWD as an assessment of functional capacity in patients with pulmonary disease is evolving, with recent data suggesting that a minimal clinically meaningful difference is $54-80 \mathrm{~m}^{23}$

Time to clinical worsening, defined as time from randomization to occurrence of death, lung transplantation, hospitalization for PAH, atrial septostomy, study withdrawal because of addition of other PAH medications, or meeting the early escape criteria, was also assessed. ${ }^{21}$ In order to be classified as "time to clinical worsening due to early escape criteria" the patient had to meet two of the following criteria: $a>20 \%$ decrease in 6MWD, an increase in WHO functional classification, worsening right ventricular failure, progressing hepatic or renal failure, or systolic blood pressure $<85 \mathrm{mmHg}$.

Time to clinical worsening was significantly better in the ambrisentan group, but the observed benefit was not dose-dependent. ${ }^{21}$ Patients receiving the $2.5 \mathrm{mg}$ or $5 \mathrm{mg}$ dose showed similar benefits $(P=0.005)$, with no statistically significant benefit $(P=0.214)$ observed in patients receiving the $10 \mathrm{mg}$ dose. The most common cause of clinical worsening was hospitalization for PAH.

ARIES-1 demonstrated a significant improvement in WHO functional classification for patients receiving ambrisentan as compared with placebo $(P=0.036)$, whereas ARIES-2 demonstrated similar improvements in WHO functional classification but did not achieve statistical significance $(P=0.117) .{ }^{21}$ The observed benefit in terms of improvement 
in functional classification was due primarily to the treatment effect on functional classification deterioration, which was significantly lower in patients receiving ambrisentan as compared with placebo (ARIES-1, 3.0\% versus 16.4\%; ARIES-2, 3.9\% versus $18.5 \%$ ).

Plasma B-type natriuretic peptide is a prognostic factor that is inversely correlated with functional capacity, and elevated levels are associated with increased mortality. ${ }^{24}$ Plasma B-type natriuretic peptide levels were reported as percent increases and decreases from baseline in the placebo and ambrisentan groups. ${ }^{21}$ A slight increase in baseline plasma B-type natriuretic peptide levels was reported in patients receiving placebo in the ARIES-1 (9\%) and ARIES-2 (13\%) studies. In contrast, a significant decrease in plasma B-type natriuretic peptide levels (29\%, 30\%, and 45\%) was reported in patients receiving ambrisentan $2.5 \mathrm{mg}, 5 \mathrm{mg}$, and $10 \mathrm{mg}$, respectively.

A significant improvement in patient quality of life as measured by the Short Form-36 Health Survey Physical Functioning scale was reported by ARIES-2 $(P=0.005) .{ }^{21}$ A similar trend in quality of life improvement was noted in the ARIES-1 study, but was not statistically significant.

\section{ARIES-E}

The ARIES-E study was an extension protocol of the ARIES-1 and ARIES-2 studies, in which all patients who completed the studies, or dropped out due to the early escape criteria, were eligible to continue monotherapy with ambrisentan for an additional two years. ${ }^{25}$

A total of 383 patients were enrolled in the ARIES-E protocol and are included in the data analysis. ${ }^{25}$ Patients who were assigned to an active treatment as part of ARIES-1 and ARIES-2 were allowed to continue their originally assigned doses, and those who were receiving placebo were blindly randomized in a $1: 1: 1$ ratio to receive $2.5 \mathrm{mg}, 5 \mathrm{mg}$, or $10 \mathrm{mg}$ of ambrisentan.

Efficacy, as assessed by the 6MWD, Borg dyspnea index, WHO functional class, long-term survival, and time to clinical worsening, was evaluated at baseline, at 4, 12, 16, 24, 36, and 48 weeks, and at 24 -week intervals thereafter. ${ }^{25}$

Significant improvements in exercise capacity, as measured by the 6MWD, were observed in patients taking ambrisentan $5 \mathrm{mg}$ (28 m; 95\% CI 14-42) and $10 \mathrm{mg}$ (37 m; 95\% CI 22-52) after one year of therapy. ${ }^{25}$ This benefit was maintained after two years of therapy, with patients taking $5 \mathrm{mg}$ and $10 \mathrm{mg}$ improving by an average $23 \mathrm{~m} \mathrm{(95 \%} \mathrm{CI}$ 9-38) and $28 \mathrm{~m}$ (95\% CI 11-45), respectively. Patients taking ambrisentan $2.5 \mathrm{mg}$ daily showed improvements in exercise capacity after one year (25 m; 95\% CI 5-45), but the benefit was not sustained after two years of therapy $(21 \mathrm{~m}$; $95 \%$ CI -2 to 45$)$.

Similarly, an improvement in the Borg dyspnea index was observed in patients taking $5 \mathrm{mg}(-0.59 ; 95 \% \mathrm{CI}-0.94$ to -0.23$)$ or $10 \mathrm{mg}(-0.51 ; 95 \% \mathrm{CI}-1.00$ to -0.03$)$ of ambrisentan after one year of therapy. ${ }^{25}$ This benefit was sustained after two years of therapy in patients receiving ambrisentan $10 \mathrm{mg}(-0.60 ; 95 \% \mathrm{CI}-1.08$ to -0.11$)$. A trend towards sustained improvement after two years of therapy was observed in the $5 \mathrm{mg}$ group $(-0.33 ; 95 \% \mathrm{CI}-0.68$ to 0.03 ), but was not statistically significant. No benefit on the Borg dyspnea index was observed in the $2.5 \mathrm{mg}$ group after one $(-0.08 ; 95 \% \mathrm{CI}-0.55$ to 0.38$)$ or two $(0.23$; $95 \% \mathrm{CI}-0.31$ to 0.76$)$ years of therapy.

Long-term survival was analyzed for the overall patient population and for idiopathic PAH and PAH associated with connective tissue disease subgroups. ${ }^{24}$ The reported survival rates were significantly higher than those estimated by the National Institute of Health Registry (one-year survival $72 \%$ and two-year survival $61 \%$ ) in the combined treatment groups, 94\% (95\% CI 91-96) and 88\% (95\% CI 83-91) at one and two years, respectively. There were no differences in survival between the three treatment groups. Similar survival rates were also observed in the subgroups diagnosed with idiopathic PAH (96\% at year 1 and $89 \%$ at year 2) and those having PAH associated with a connective tissue disorder (91\% at year 1 and $83 \%$ at year 2 ).

Time to clinical worsening, as previously defined by the criteria found in ARIES-1 and ARIES-2 with the additional criteria of "add-on prostacyclin analog therapy", was assessed throughout the study. ${ }^{25}$ The most common causes of clinical worsening were hospitalization $(n=76)$, death $(n=42)$, and addition of prostacyclin therapy $(n=34)$. After one and two years of treatment, the overall population free from clinical worsening was 83\% (95\% CI 79-87) and 72\% (95\% CI 67-76), respectively, with similar findings across the ambrisentan dosing groups.

Other benefits such as improvements in mean pulmonary arterial pressure, cardiac output, pulmonary vascular resistance, and right ventricular ejection fraction have also been reported. In a retrospective analysis completed at one clinical site, the hemodynamic parameters of 12 patients participating in the ARIES-1 and ARIES-E trials were analyzed. ${ }^{26}$ Significant improvements were reported in mean pulmonary arterial pressure, cardiac output, and pulmonary vascular resistance at one year $\left(P=0.02, P=0.03, P<0.01\right.$, respectively) ${ }^{26}$ An increase in right ventricular ejection fraction was also 
reported (29\% at baseline versus $46 \%$ at 2 years; $P=0.02) .{ }^{26}$ Although this study is not large enough to generalize the findings to all patients with $\mathrm{PAH}$, it does provide a clinically meaningful insight into the potential impact ambrisentan can have on pulmonary and cardiac hemodynamics.

\section{ARIES-3}

The ARIES-3 study was a long-term, open-label, single-arm, multicenter study that assessed the efficacy and safety of ambrisentan in patients with PAH from WHO groups 1, 3, 4 , and $5 .{ }^{27} \mathrm{~A}$ total of 224 patients were enrolled in the study, and received $5 \mathrm{mg}$ of ambrisentan for 24 weeks. Unlike ARIES-1 and ARIES-2, patients were not excluded if they were receiving any of the following chronic PAH therapies: intravenous epoprostenol, treprostinil, inhaled iloprost, or sildenafil. Patients were also included if they had failed therapy with bosentan or sitaxsentan because of a serum aminotransferase concentration greater than three times the upper level of normal, as long as serum aminotransferase concentrations were less than three times the upper level of normal at the time of screening.

The primary endpoint of the study was change from baseline in functional exercise capacity as measured by the $6 \mathrm{MWT}^{27}{ }^{27}$ Secondary endpoints were evaluated for changes from baseline and included: plasma B-type natriuretic peptide, WHO functional classification status, and Borg dyspnea scores. Patient enrolled in the study were prominently WHO functional classification II (29\%) and III (65\%), and $53 \%$ were receiving chronic $\mathrm{PAH}$ therapy at the time of enrollment.

Exercise capacity was increased significantly from baseline at 24 weeks by $21 \mathrm{~m}(95 \%$ CI $12-29, P<0.001) .{ }^{27}$ Exercise capacity was also significantly higher in patients receiving background therapy with sildenafil $(25 \mathrm{~m} ; 95 \% \mathrm{CI}$ 11-40), prostacyclins (46 m; 95\% CI 7-85), and ambrisentan monotherapy (32 m; 95\% CI 17-48). Plasma B-type natriuretic peptide concentrations decreased by $26 \%$ (95\% CI 16-34), dyspnea (assessed by the Borg dyspnea index) was decreased by 0.5 (95\% CI $0.3-0.8)$, and WHO functional classification was significantly improved $(P<0.001)$ at 24 weeks of treatment.

\section{Assessment of safety ARIES-I and ARIES-2}

Ambrisentan was generally well tolerated in all patient groups, with a similar number of patients discontinuing placebo $(n=21)$ and ambrisentan $(n=20)$ due to adverse drug events, worsening PAH, or because they met an early escape criterion. ${ }^{21}$ Patients receiving ambrisentan reported significantly more peripheral edema, headache, and nasal congestion (Table 2). These side effects were considered mild to moderate in nature. None of the patients randomized to ambrisentan experienced an elevation of serum aminotransferase concentrations greater than three times the upper limit of normal. Similarly, mean values for total bilirubin, alanine aminotransferase, aspartate aminotransferase, and alkaline phosphate did not increase from baseline. Ambrisentan did not have a clinically significant effect on anticoagulation, but was associated with a decrease in mean hemoglobin concentration $(0.84 \mathrm{~g} / \mathrm{dL} \pm 1.2 \mathrm{~g} / \mathrm{dL})$.

\section{ARIES-E}

Safety, as assessed by adverse event monitoring, vital signs, and physical examination, was evaluated at baseline, at $4,12,16,24,36$, and 48 weeks, and at 24-week intervals thereafter. ${ }^{25}$ Clinical laboratory tests and urine pregnancy tests for female patients were assessed every four weeks.

The adverse drug events reported by ARIES-E are consistent with the findings of the ARIES-1 and ARIES-2 studies. Peripheral edema, headache, respiratory tract infection, and dizziness were the most commonly reported adverse drug reactions and were mild to moderate in intensity. ${ }^{25}$ The most commonly reported reasons for discontinuation of therapy or death during treatment were right ventricular failure $(3.9 \%)$, worsening PAH (3.7\%), and acute respiratory failure $(1.0 \%)$. The frequency with which adverse drug reactions occurred

Table 2 Most frequent adverse events in ARIES-I and ARIES-2 ${ }^{17}$

\begin{tabular}{|c|c|c|c|c|c|}
\hline \multirow[t]{2}{*}{ Adverse event, n (\%) } & \multirow{2}{*}{$\begin{array}{l}\text { Placebo } \\
n=132\end{array}$} & \multicolumn{4}{|c|}{ Ambrisentan } \\
\hline & & $\begin{array}{l}2.5 \mathrm{mg} \\
\mathrm{n}=64\end{array}$ & $\begin{array}{l}5 \mathrm{mg} \\
\mathrm{n}=130\end{array}$ & $\begin{array}{l}10 \mathrm{mg} \\
\mathrm{n}=67\end{array}$ & $\begin{array}{l}\text { Combined } \\
n=26 I\end{array}$ \\
\hline Subjects with $\geq I A E$ & $108(81.8)$ & $47(73.4)$ & $102(78.5)$ & $53(79.1)$ & $202(77.4)$ \\
\hline Peripheral edema & $14(10.6)$ & $2(3.1)$ & $24(18.5)$ & $19(28.4)$ & $45(17.2)$ \\
\hline Headache & $18(13.6)$ & $5(7.8)$ & $20(15.4)$ & $13(19.4)$ & $28(14.6)$ \\
\hline Dizziness & $13(9.8)$ & $3(4.7)$ & $9(6.9)$ & $6(9.0)$ & $18(6.9)$ \\
\hline Nasal congestion & $2(1.5)$ & I (I.6) & $7(5.4)$ & $7(10.4)$ & $15(5.7)$ \\
\hline
\end{tabular}

Abbreviations: AE, adverse event; ARIES, Ambrisentan in Pulmonary Arterial Hypertension, Randomized, Double-Blind, Placebo-Controlled, Multicenter Efficacy Studies. 
was similar in all three dosing groups, with the exception of worsening $\mathrm{PAH}$, which occurred more frequently in the $2.5 \mathrm{mg}$ group (7.3\%) than the $5 \mathrm{mg}(2.1 \%)$ and $10 \mathrm{mg}(3.1 \%)$ treatment groups.

The risk of developing alanine and/or aspartate aminotransferase levels greater than three times the upper limit of normal was relatively low, occurring in $1.8 \%$ and $3.9 \%$ of patients at year 1 and year 2, respectively. Only two patients discontinued ambrisentan as a result of hepatic enzyme elevation. ${ }^{25}$ In addition, ambrisentan was associated with a reduction in mean hemoglobin levels at week $4(0.9 \mathrm{~g} / \mathrm{dL})$ and week $108(1.2 \mathrm{~g} / \mathrm{dL}) .{ }^{25}$ Ambrisentan had no effect on control of anticoagulation.

\section{ARIES-3}

Similar adverse drug events to those reported in the ARIES-1, ARIES-2, and ARIES-E studies were seen in the ARIES-3 study. ${ }^{27}$ The most frequent side effects reported included peripheral edema, headache, dyspnea, upper respiratory tract infection, and nasal congestion. These side effects were mild to moderate in nature, and rarely resulted in discontinuation of therapy. Some serious side effects were reported, including right ventricular heart failure, pneumonia, and worsening PAH. This was not a placebo-controlled study, so it is difficult to assess whether these adverse events were as a result of disease progression or use of ambrisentan.

Supporting the findings of the previously discussed studies, ARIES-3 did not report a significant increase in aminotransferase levels in patients receiving ambrisentan. ${ }^{27}$ Four patients had alanine and/or aspartate aminotransferase levels that were greater than three times the upper limit of normal but less than five times the upper limit of normal, and were allowed to continue therapy with no adverse outcomes reported. Two patients experienced alanine and/or aspartate aminotransferase levels that were greater than eight times the upper limit of normal and were not allowed to continue therapy; one patient was diagnosed with autoimmune hepatitis and the second patient was found to be taking other hepatotoxic drugs. Further, the mean hemoglobin concentration showed a slight decrease from baseline at 24 weeks $(0.92 \pm 1.3 \mathrm{~g} / \mathrm{dL})$, but was not correlated with any major bleeding events.

\section{Patient acceptability}

Currently, there are no clinical studies evaluating patient acceptability of ambrisentan therapy. Ambrisentan has a relatively benign safety profile, with the most common side effects of peripheral edema, headache, and nasal congestion being mild to moderate in nature..$^{21,25,27}$ Patient compliance and acceptability of ambrisentan was not significantly affected by side effects, and rarely led to discontinuation in the ARIES-1, ARIES-2, and ARIES-3 studies. ${ }^{21,27}$

As compared with bosentan, ambrisentan has several favorable qualities that potentially make it more acceptable to patients. Ambrisentan is administered once daily, compared with bosentan, which is administered twice daily. This may result in improved adherence, because a decrease in dosing frequency has been associated with improved medication adherence in patients with chronic disease. ${ }^{28}$

There is minimal risk of liver enzyme elevation associated with ambrisentan, and it can be used safely in high-risk patients who have previously failed bosentan or sitaxsentan therapy because of liver enzyme abnormalities. ${ }^{29}$ Ambrisentan does not require monthly liver aminotransferase tests to assess and monitor for risk of liver injury. This requirement was removed in March 2011 after the FDA revised the black box warning to exclude liver injury. ${ }^{30}$ The FDA cited the low incidence of liver injuries in both clinical trial and post-marketing studies to support their decision. ${ }^{30}$ The FDA currently recommends that providers order a liver function test as clinically necessary in patients using ambrisentan. ${ }^{30}$

Further, because it is only a minor CYPC19 and CYP3A4 substrate, ambrisentan has a more favorable metabolism and drug interaction profile than bosentan (Table 1). ${ }^{16,31}$ Ambrisentan is safe for use with all PAH and most non-PAH therapies, with cyclosporine being the only established drug-drug interaction. ${ }^{16}$ In contrast, bosentan is both a major substrate and inducer of CYP2C9 and CYP3A4, so has the potential to interact with numerous medications, including several agents used to treat PAH (sildenafil, tadalafil, nifedipine, warfarin). ${ }^{31}$

Ambrisentan and bosentan have both been associated with decreases in hemoglobin concentration. ${ }^{16,31}$ This has not been associated with an increased risk of hemorrhage or hemolysis, but anemia has been reported during post-marketing surveillance. ${ }^{16,31}$ Use of ambrisentan is not contraindicated in patients with decreased hemoglobin levels, but is not recommended for use in those with clinically significant anemia. ${ }^{16}$ The recommended monitoring is slightly different between the two agents, with bosentan requiring more frequent monitoring than ambrisentan. ${ }^{16,31}$

Other factors that may influence patient acceptability include the reproductive risks associated with endothelin receptor antagonists. One such risk is the potential for decreased spermatogenesis in males. Currently, this adverse effect has only been reported with the use of bosentan, but 
the risk cannot be excluded for ambrisentan. ${ }^{16,31}$ Further, the potential risk of teratogenicity associated with this drug class may be concerning to patients. However, this risk is extensively monitored through the comprehensive Risk Evaluation and Mitigation Strategies programs. While there are no comparative studies between the endothelin receptor antagonists and phosphodiesterase type 5 inhibitors (sildenafil, tadalafil), clinicians may be inclined to use phosphodiesterase type 5 inhibitors because of their ready availability and favorable adverse effect profile.

\section{Conclusion}

Ambrisentan is a selective $\mathrm{ET}_{\mathrm{A}}$ receptor antagonist approved by the FDA for treatment of patients with PAH WHO group 1. Ambrisentan is effective at increasing exercise tolerance, decreasing the risk of functional class deterioration, prolonging time to clinical worsening, and increasing patient survival rates. ${ }^{21,25,27}$ Treatment with ambrisentan is generally well tolerated, and side effects associated with its use are considered mild to moderate in nature (peripheral edema, headache, sinusitis, flushing, and nasal congestion). ${ }^{21,25,26}$ Ambrisentan has several favorable qualities that potentially make it more acceptable to patients as compared with bosentan therapy including: once-daily administration, limited adverse drug reactions, few drug-drug interactions, and minimal risk of hepatic enzyme elevation. Access to ambrisentan is restricted through LEAP, because of the potential risk of teratogenicity associated with its use. As with many of the current treatments of $\mathrm{PAH}$, further clinical trials are needed to define the optimal utilization of ambrisentan for treatment of the disease.

\section{Disclosure}

The authors report no conflicts of interest in this work.

\section{References}

1. D’Alonzo GE, Barst RJ, Ayres SM, et al. Survival in patients with primary pulmonary hypertension: results from a national prospective registry. Ann Intern Med. 1991;115:343-349.

2. McGoon M, Gutterman D, Steen V, et al. Screening, early detection, and diagnosis of pulmonary arterial hypertension: ACCP evidence-based clinical practice guidelines. Chest. 2004;126:14S-34S.

3. Simonneau G, Galiè N, Rubin LJ, et al. Clinical classification of pulmonary hypertension. J Am Coll Cardiol. 2004;43(12 Suppl S): $5 \mathrm{~S}-12 \mathrm{~S}$

4. Galie N, Hoeper MM, Humbert M, et al. Guidelines for the diagnosis and treatment of pulmonary hypertension: the Task Force for the Diagnosis and Treatment of Pulmonary Hypertension of the European Society of Cardiology (ESC) and the European Respiratory Society (ERS). Eur Heart J. 2009;30:2493-2537.

5. Farber HW, Loscalzo J. Pulmonary arterial hypertension. N Engl J Med. 2004;351:1655-1665.
6. Giaid A, Yanagisawa M, Langleben D, et al. Expression of endothelin-1 in the lungs of patients with pulmonary hypertension. N Engl J Med. 1993;328:1732-1739.

7. Inoue AM, Yanagisawa S, Kimura Y, et al. The human endothelin family: three structurally and pharmacologically distinct isopeptides predicted by three separate genes. Proc Natl Acad Sci U S A. 1989;86:2863-2867.

8. Yanagisawa M, Kurihara H, Kimura S, et al. A novel potent vasoconstrictor peptide produced by vascular endothelial cells. Nature. 1988;332:411-415.

9. Rubanyi GM, Polokoff PM. Endothelins: molecular biology, biochemistry, pharmacology, physiology, and pathophysiology. Pharmacol Rev. 1994;46:325-415.

10. Davie N, Haleen SJ, Upton PD, et al. ET(A) and ET(B) receptors modulate the proliferation of human pulmonary artery smooth muscle cells. Am J Respir Crit Care Med. 2002;165:398-405.

11. Alberts GF, Peifley KA, Johns A, Kleha JF, Winkles JA. Constitutive endothelin-1 overexpression promotes smooth muscle cell proliferation via an external autocrine loop. J Biol Chem. 1994;269:10112-10118.

12. Fukuroda T, Fujikawa T, Ozaki S, et al. Clearance of circulating endothelin-1 by ETB receptors in rats. Biochem Biophys Res Commun. 1994;199:1461-1465.

13. Fukuroda T, Kobayashi M, Ozaki S, et al. Endothelin receptor subtypes in human versus rabbit pulmonary arteries. J Appl Physiol. 1994;76:1976-1982.

14. Pfizer initiates product withdrawal of THELIN ${ }^{\circledR}$ (sitaxsentan sodium) worldwide. Available from: http://www.hc-sc.gc.ca/dhp-mps/alt_ formats/pdf/medeff/advisories-avis/prof/2010/thelin_2_hpc-cps-eng. pdf. Accessed February 26, 2013.

15. Anderson JR, Nawarskas JJ. Pharmacotherapeutic management of pulmonary arterial hypertension. Cardiol Rev. 2010;18:148-162.

16. Letairis [package insert]. Foster City, CA: Gilead Sciences Inc; Oct 2012. Available from: http://www.gilead.com/pdf/letairis_pi.pdf. Accessed November 24, 2012.

17. US Food and Drug Administration. Drug approval package: Letairis (ambrisentan NDA \#022081). Medical Review, Part 1. Available from: http://www.accessdata.fda.gov/drugsatfda_docs/ nda/2007/022081s000_MedR_P1.pdf. Accessed November 11, 2012.

18. Galie N, Badesch D, Oudiz R, et al. Ambrisentan therapy for pulmonary arterial hypertension. J Am Coll Cardiol. 2005;46:529-535.

19. Gilead Sciences Inc. Prescriber Guide: Letairis and the LEAP program. Foster City, CA: Gilead Sciences Inc; Feb 2012.

20. Blankart CR, Stargardt T, Schreyögg J. Availability of and access to orphan drugs: an international comparison of pharmaceutical treatments for pulmonary arterial hypertension, Fabry disease, hereditary angioedema and chronic myeloid leukaemia. Pharmacoeconomics. 2011;29:63-82.

21. Galiè N, Olschewski H, Oudiz RJ, et al. Ambrisentan for the treatment of pulmonary arterial hypertension: results of the ambrisentan in pulmonary arterial hypertension, randomized, double-blind, placebocontrolled, multicenter, efficacy (ARIES) study 1 and 2. Circulation. 2008;117:3010-3019.

22. Salzman SH. The 6-min walk test: clinical and research role, technique, coding, and reimbursement. Chest. 2009;135:1345-1352.

23. Wise RA, Brown CD. Minimal clinically important differences in the six-minute walk test and the incremental shuttle walking test. COPD. 2005;2:125-129.

24. Nagaya N, Nishikimi T, Uematsu M, et al. Plasma brain natriuretic peptide as a prognostic indicator in patients with primary pulmonary hypertension. Circulation. 2000;102:865-870.

25. Oudiz RJ, Galiè N, Olschewski H, et al. Long-term ambrisentan therapy for the treatment of pulmonary arterial hypertension. J Am Coll Cardiol. 2009;54:1971-1981.

26. Blalock SE, Matulevicius S, Mitchell LC, et al. Long-term outcomes with ambrisentan monotherapy in pulmonary arterial hypertension. J Card Fail. 2010;16:121-127. 
27. Badesch DB, Feldman J, Keogh A, et al. ARIES-3: ambrisentan therapy in a diverse population of patients with pulmonary hypertension. Cardiovasc Ther. 2012;30:93-99.

28. Coleman CI, Limone B, Sobieraj DM, et al. Dosing frequency and medication adherence in chronic disease. J Manag Care Pharm. 2012;18:527-539.

29. McGoon MD, Frost AE, Oudiz RJ, et al. Ambrisentan therapy in patients with pulmonary arterial hypertension who discontinued bosentan or sitaxsentan due to liver function test abnormalities. Chest. 2009;135:122-129.
30. US Food and Drug Administration. FDA drug safety communication: liver injury warning to be removed from Letairis (ambrisentan) tablets. Available from: http://www.fda.gov/Drugs/DrugSafety/ucm245852. htm. Updated 01/10/2012. Accessed September 26, 2012.

31. Tracleer [package insert]. South San Francisco, CA: Actelion Pharmaceuticals US Inc; October 2012. Available from: http://www.tracleer. com/docs/Tracleer_Full_Prescribing_Information.pdf. Accessed December 11, 2012.

\section{Publish your work in this journal}

Patient Preference and Adherence is an international, peer-reviewed, open access journal focusing on the growing importance of patient preference and adherence throughout the therapeutic continuum. Patient satisfaction, acceptability, quality of life, compliance, persistence and their role in developing new therapeutic modalities and compounds to optimize clinical outcomes for existing disease states are major areas of interest. This journal has been accepted for indexing on PubMed Central. The manuscript management system is completely online and includes a very quick and fair peer-review system. Visit http://www.dovepress.com/ testimonials.php to read real quotes from published authors.

Submit your manuscript here: http://www.dovepress.com/patient-preference-and-adherence-journal 